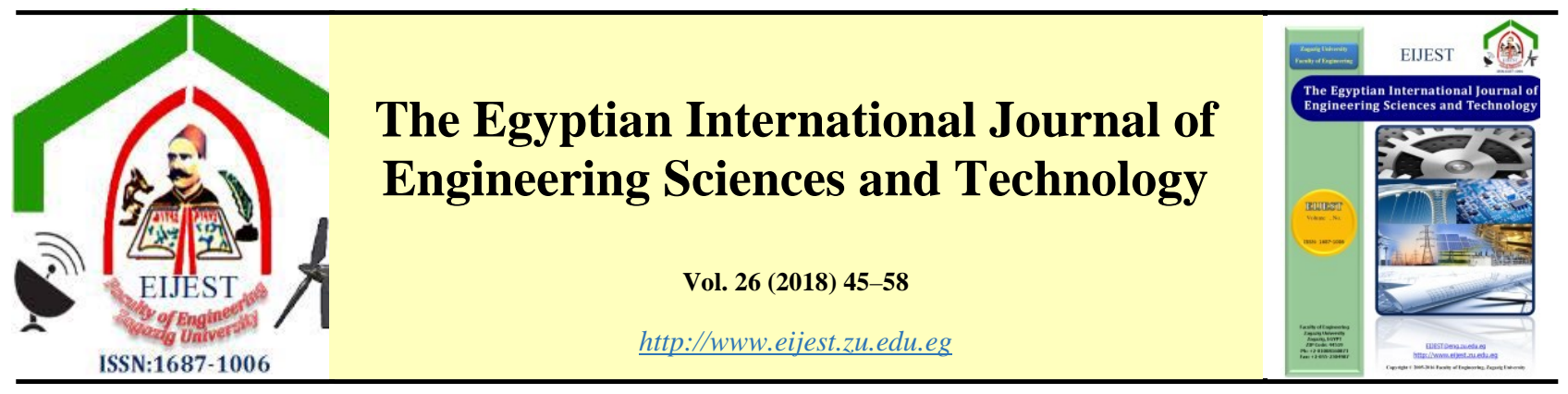

\title{
Vibration Signature of Unbalanced Rotors of Centrifugal Pumps
}

\author{
Ahmed Abd-Elbar Salman, Ahmed H. Osman*, Khaled M. Fawzy \\ Zagazig University, Zagazig, 44519, Egypt
}

\begin{tabular}{l}
\hline A R T I C L E I N FO \\
\hline Article history: \\
Received 11 July 2018 \\
Received in revised form \\
07 November 2018 \\
Accepted 29November \\
2018 \\
Available online 03 \\
December2018 \\
\hline
\end{tabular}

Keywords:

Centrifugal Pump

Unbalanced Rotors

FFT

Vibration Signature

Vibration Analysis

\begin{abstract}
A B S T R A CT
Centrifugal pumps are widely used in many industrial applications. It is necessary to detect undesired conditions during the operation of it to avoid consequential damages. Early detection is a vital factor to increase the availability and reliability. Vibration analysis is an effective and efficient tool in diagnosing rotating equipment. Vibration signature of unbalanced rotors needs to investigate different running conditions of the pump. The increase in the vibration level indicates a probable mechanical failure. Unbalance is one of probable reason of the vibration in the machines. The unbalanced rotor always produces high vibration level and excessive loads on the area of the bearing and hence reducing the machine lifetime. The investigated system monitors a four stages centrifugal pump to expect the vibration spectrum of the unbalanced rotors. The driving motor runs at 2980 RPM with rated power $55 \mathrm{~kW}$. This pumping system uses seawater. Comparing the vibration data of the inspected pump (in normal and faulty conditions) with each other and vibration limits of ISO10816. The results from this study clarifies the importance of vibration analysis in detecting the unbalanced rotors. Experimental results show that primarily $1 \mathrm{X}$ shaft running speed with sinusoidal time waveform can characterize the unbalance in shaft. The time waveform may be pure sine wave and it may contain noise. To eliminate the noise, the user may use a suitable digital or analogue filters (i.e. software or hardware). The amplitude of vibration in all directions for faulty condition is higher than the amplitude value at $1 \mathrm{X}$ RPM frequency in the normal condition. The amplitude at the $1 \mathrm{X}$ varies proportional to the square of the rotational speed. The induced forces due to unbalance on both bearing of the rotor are always at the same direction where the vibration signal from them are in phase with eachother.
\end{abstract}

\section{Abbreviations}

$\begin{array}{llll}\text { A } & \text { Axial } & \text { MNDE } & \text { Motor Non Drive End } \\ \text { DE } & \text { Drive End } & \text { NDE } & \text { Non Drive End } \\ \text { DSS } & \text { Deflected Shape of Shaft } & \text { ODS } & \text { Operational Defect Shape } \\ \text { FFT } & \text { Fast Fourier Transformation } & \text { PDE } & \text { Pump Drive End } \\ \text { GCL } & \text { Geometric Centerline } & \text { PNDE } & \text { Pump Non Drive End } \\ \text { H } & \text { Horizontal } & \text { RMS } & \text { Root Mean Square } \\ \text { HP } & \text { High Pressure } & 1 X & 11^{\text {storder Frequency i.e Frequency/shaft }} \\ \text { MDE } & \text { Motor Drive End } & & \text { rotating Frequency } \\ \text { PIA } & \text { Principle Inertia Axis } & \text { V } & \text { Vertical }\end{array}$




\section{Introduction}

With vibration measurements, we can determine defects such as dynamic imbalance, faulty alignment, gaming and weaknesses, bearing specific defects, etc. [1]. Unbalance is one of the vibration sources in the electric motors and centrifugal pumps. There are many reasons for this problem in the electric motors and the pumps. These problems may arise due to defects during manufacturing, corrosion in the pump fan, partial permanent pollution of the pump fan blade, the unbalanced mounting of one of the pump bearings carrying the whole loads of pump fan, improper mass distribution in the rotor arms and blades of the electric motor, the bending of the pump and electric motor shaft. The imbalance problem encountered in electric motor and centrifugal pumps causes negative effects on pump efficiency [2]. Rotor unbalances may be considered as one of the most common reasons in machine's vibrations. Eliminating the mass unbalance can solve most of the problems of the rotating machinery. Excessive centrifugal forces (in the rotating component) will be generated due to the mass unbalance that may also reduce the reliability and lifetime of the mechanical component. The induced force which always causes more vibration and adds additional load on the bearing area. High speed rotating machines may suffer from severe problems due to a very small amount of unbalance. The unbalanced system of overhung rotors is taken into consideration in numerous unbalance studies. Many researchers have studied techniques of faults diagnosis related to unbalance. K. P. Ramachandran [3] presented a general method which can be used to obtain the unbalance response orbit based on the experimental, where the shafts rotate at different speeds. A single mass Experiments were carried out at five different speeds and plotted the corresponding results. The unbalanced rotor can be identified by phase analysis and spectral. Surendra N. Ganeriwal and Brian Schwarz [4], have used the operationaldeflection shape (ODS) to detect the unbalance of rotating components in the rotating machines.

V. Hariharan and P. S. S. Srinivasan [5] carried out a simulation for unbalance. The experimental data and simulation are compared with each other. Both of the experimental and simulated data show that the unbalance can be primarily characterized by first harmonic (1X) of the shaft running speed. M. Abdul Saleem and G. Diwakar [6] used the Deflected Shape of Shaft (DSS) of a rotating equipment to identify the unbalance in its rotating component where the presence of unbalance causes deflected shape shaft. Girish D. Mehta, Vijaykumar S. Shende, et al. [7] carried out the mathematical model for prediction of unbalance. They found that the amplitude at $1 \mathrm{X}$ frequency will be the confirmation of the amplitude at the unbalancing condition.

\section{Types ofUnbalance}

- Static unbalance where the Geometric Centerline (GCL) and Principle Inertia Axis (PIA) are parallel

- Couple unbalance GCL and PIA intersect inthecenter

-Dynamic unbalance GCL and PIA do not touch or coincide,

For all types of unbalance, the Fast Fourier Transform (FFT) vibration signature will show a predominant 1X RPM frequency signature. Vibration amplitude at the $1 \mathrm{X}$ RPM frequency will vary proportionally with the square of the rotational speed. This peak always exists and usually controls the vibration. Figure 1 shows the frequency response of a normal unbalance of rotating shaft.

Static unbalance will be in-phase and steady (15$20^{\circ}$ ). Looking at the vibration signal in the horizontal $(\mathrm{H})$ direction, the phase will be shifted by $90^{\circ}\left( \pm 30^{\circ}\right)$. Another test is to move the pickup from one bearing to another in the same plane (vertical or horizontal). The phase remains the same if the fault is a static unbalance. The static unbalance is shown in Figure2.

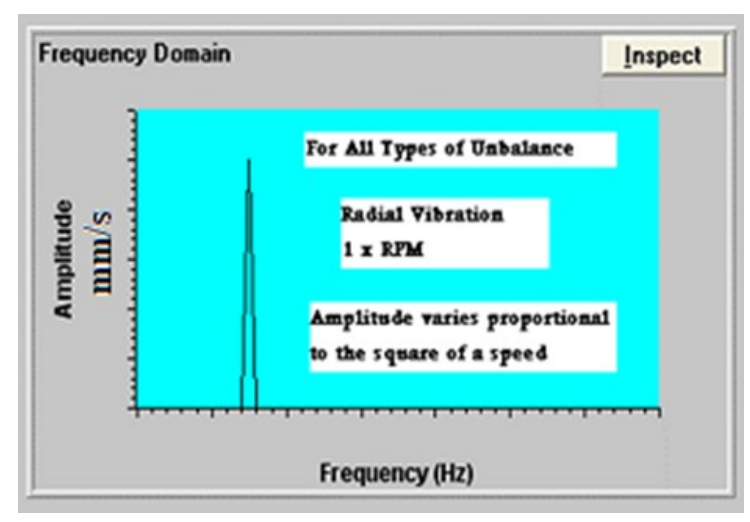

Fig. 1: Vibration signature due to unbalance 


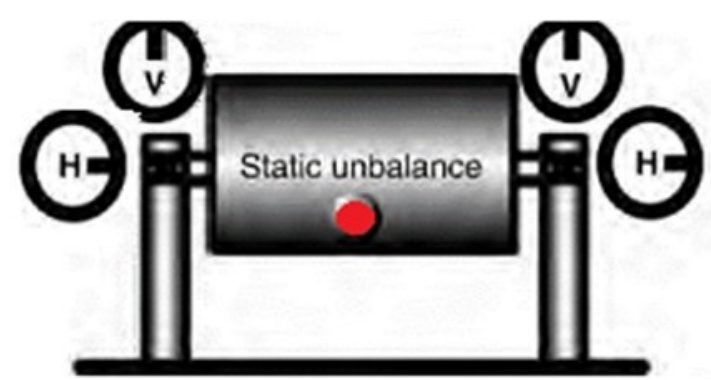

Fig. 2: Phase relationship - static unbalance

In a couple unbalance as shown in Figure 3, the FFT vibration signature again displays a single $1 \mathrm{X}$ RPM frequency peak. The amplitude at the $1 \mathrm{X}$ varies proportionally with the square of rotational speed. This defect may cause high axial and radial vibrations. Couple unbalance tends to be $180^{\circ}$ out of phase on the same shaft. Note that, almost a $180^{\circ}$ phase difference exists between two bearings in the horizontal plane. The same is observed in the vertical plane. It is advisable to perform an Operational Deflection Shape (ODS) analysis to check if couple unbalance exists in the system or not.

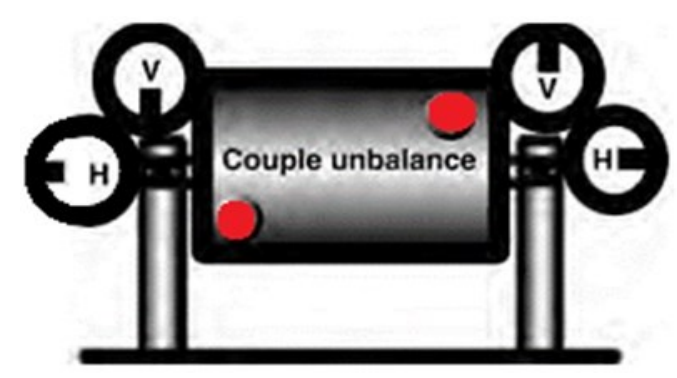

Fig. 3: Phase relationship - couple unbalance

In case of overhung rotors, the FFT vibration signature displays a single $1 \mathrm{X}$ RPM peak as well, and the amplitude again varies proportionally with the square of the rotational speed. High axial and radial vibrations may arise in this case. The axial phase on the two bearings seems to be in phase whereas the radial phase tends to be unsteady. Overhung rotors have both static and couple unbalance. The rotor must be tested and fixed using analyzers or balancing equipment as shown in Figure4.

\section{Experimental setup}

Figure 5a shows the High Pressure (HP) pump 201, which is used in this study. Figure 5b show the schematic drawing of the pumping system and the location of the measuring sensors. In this case, the measurement carried out at both normal and unbalanced conditions in the three directions (A, V, and $\mathrm{H}$ ) simultaneously. An electric motor drives the pump. The pump has one stage to deliver oil. The motor speed is $2980 \mathrm{RPM}$ and its rated power is $55 \mathrm{~kW}$.

The data acquisition system consists of a Data Collector and Analyzer. The analyzer type is DLI Watchman. DCA-31 ${ }^{\mathrm{TM}} \mathrm{Br}$ Coupled with Expert ALERT $^{\mathrm{TM}}$ software with two-channel configurations. The sensor is an accelerometer type ICP (Integrated Circuit Piezoelectric). The sensor measures the vibration signals in the three directions (axial, horizontal and vertical at four points for the pump and the electric motor. Figure 5.b shows the location of the measuring points (pump DE bearing 3), (pump NDE bearing 4), (motor DE bearing 2) and (motor NDE bearing 1). The accelerometer is mounted on bearing of the pump and motor. The accelerometer sends the signal to the conditioning unit (DLI Watchman $\left.{ }^{\circledR D C A}-31^{\mathrm{TM}} \mathrm{Br}\right)$. The vibration signal in digital form is fed to the computer through a USB port. The software "Expert ALERT"M" reads and records the signal from the signal-conditioning unit. The Fast Fourier Transform (FFT) converts the time domain signal to the frequencydomain.

We use the ISO 10816 as a standard vibration readings for both faulty and normal operation are taken on the bearings where the defects are identified by finding the difference in amplitudes. Overall vibration levels were measured and analyzed at the normal and faulty conditions at different periods and compared with ISO 10816 [7] see Appendix. (1).

\section{Results}

\subsection{Normal condition}

The frequency vibration signature of the health pump is recorded as a baseline data for future analysis. 
These signatures are shown in Figures 6 through 9. The signals are measured at three directions (axial $\mathrm{A}$, vertical $\mathrm{V}$ and horizontal $\mathrm{H}$ ) for the pump and motor's bearings. These figures show normal overall reading for all bearings. Table 1 contains the measured amplitude readings of the normal and faulty operations at the four positions of the system (Motor Non-Drive End NDE, Motor Drive End DE, Pump DE and Pump NDE) at the three axes. These readings are compared with the alarm and critical limits.

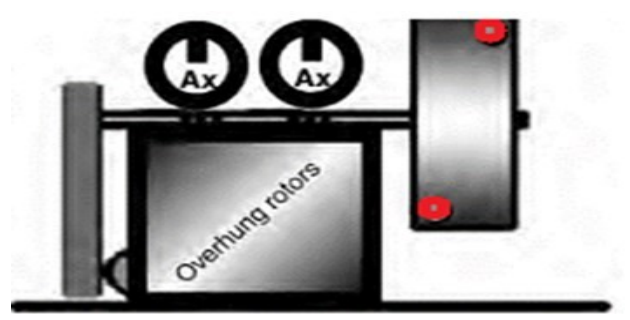

Fig. 4: Belt-driven fan/blower with an overhung rotor- The phase is measured in the axial direction

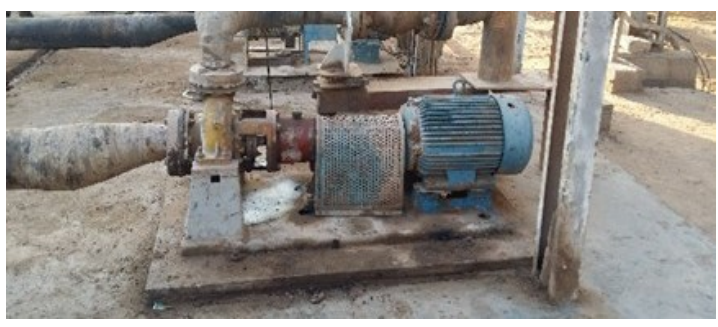

Fig. 5a: HP water pump201
Figure 6 shows the spectrum of the motor DE. It is obvious that the amplitude $(0.98 \mathrm{~mm} / \mathrm{s})$ in the vertical direction at $(1 \mathrm{X})$ is the highest amplitude.

Figure 7 shows the spectrum of the motor NDE. It is visible that the amplitude $(0.85 \mathrm{~mm} / \mathrm{s})$ in the vertical direction at $(1 \mathrm{X})$ is the highest amplitude.

Figure 8 shows the spectrum of the pump DE. It's obvious that the amplitude $(1.03 \mathrm{~mm} / \mathrm{s})$ in the vertical direction at (1X) is the highest amplitude.

Figure 9 shows the spectrum of the pump NDE. It is noticeably that the amplitude $(0.58 \mathrm{~mm} / \mathrm{s})$ in the axial direction at $(1 \mathrm{X})$ is the highest amplitude. It's obvious that Figures 6, 7, 8, and 9 represent a normal case because the readings are below the alarm limits.

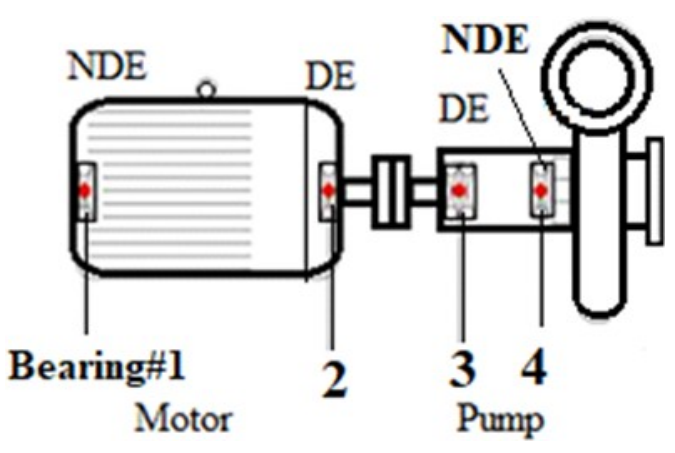

Fig. 5b: Schematic drawing for Figure 5a

Table 1. The normal and the unbalanced (faulty) overall reading

\begin{tabular}{l|lll|lll|lll|lll|l|}
\hline & \multicolumn{3}{|c|}{ Motor NDE } & \multicolumn{3}{c|}{ Motor DE } & \multicolumn{3}{c|}{ Pump DE } & \multicolumn{3}{c|}{ Pump NDE } \\
\hline Direction & $\mathrm{V}$ & $\mathrm{H}$ & $\mathrm{A}$ & $\mathrm{V}$ & $\mathrm{H}$ & $\mathrm{A}$ & $\mathrm{V}$ & $\mathrm{H}$ & $\mathrm{A}$ & $\mathrm{V}$ & $\mathrm{H}$ & $\mathrm{A}$ \\
\hline Unit & $\mathrm{mm} / \mathrm{s}$ & $\mathrm{mm} / \mathrm{s}$ & $\mathrm{mm} / \mathrm{s}$ & $\mathrm{mm} / \mathrm{s}$ & $\mathrm{mm} / \mathrm{s}$ & $\mathrm{mm} / \mathrm{s}$ & $\mathrm{mm} / \mathrm{s}$ & $\mathrm{mm} / \mathrm{s}$ & $\mathrm{mm} / \mathrm{s}$ & $\mathrm{mm} / \mathrm{s}$ & $\mathrm{mm} / \mathrm{s}$ & $\mathrm{mm} / \mathrm{s}$ \\
& RMS & RMS & RMS & RMS & RMS & RMS & RMS & RMS & RMS & RMS & RMS & RMS \\
\hline $\begin{array}{l}\text { Normal } \\
\text { Unbalanced }\end{array}$ & 1.31 & 1.18 & 1.25 & 1.35 & 1.19 & 0.898 & 2.02 & 1.43 & 1.7 & 0.951 & 0.795 & 1.22 \\
$\begin{array}{l}\text { (Faulty) } \\
\text { Alarm }\end{array}$ & 4.02 & 4.29 & 5.74 & 2.84 & 3.68 & 4.97 & 2.02 & 1.43 & 1.7 & 0.951 & 0.795 & 1.22 \\
Critical & 4.5 & 4.5 & 4.5 & 4.5 & 4.5 & 4.5 & 4.5 & 4.5 & 4.5 & 4.5 & 4.5 & 4.5 \\
\hline
\end{tabular}




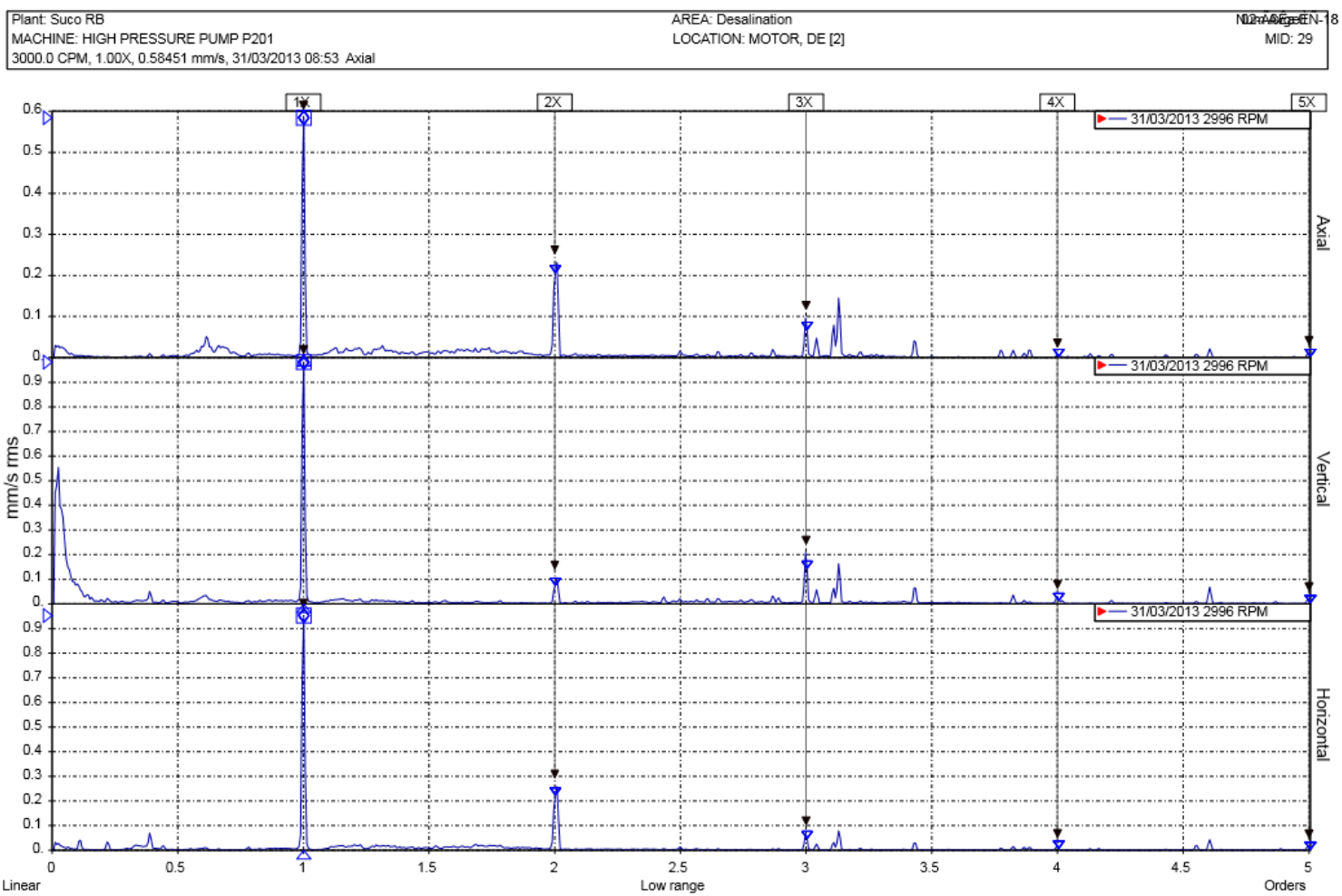

Fig. 6: Normal vibration signature of motor drive end

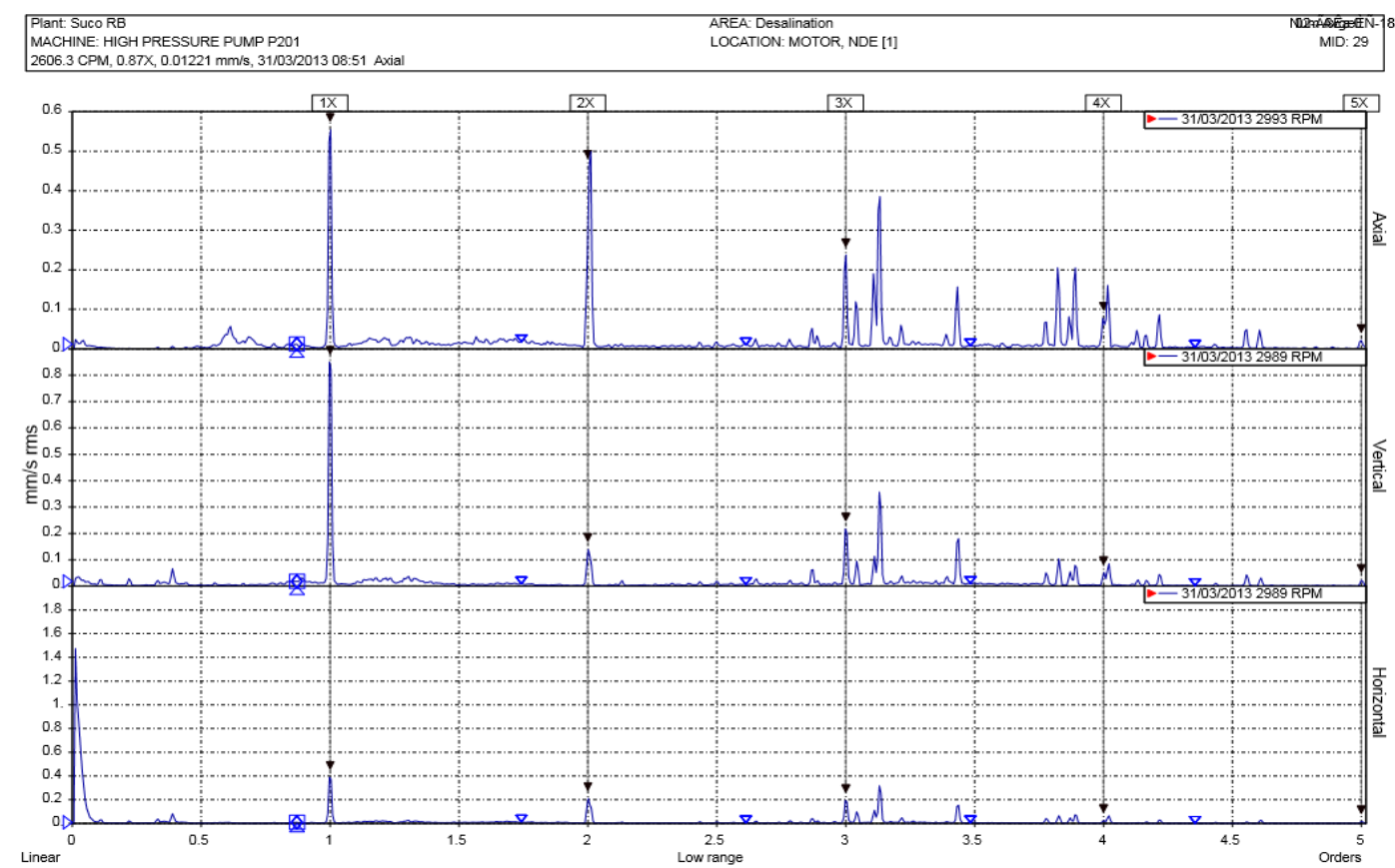

Fig. 7: Normal vibration signature of motor non-drive end 


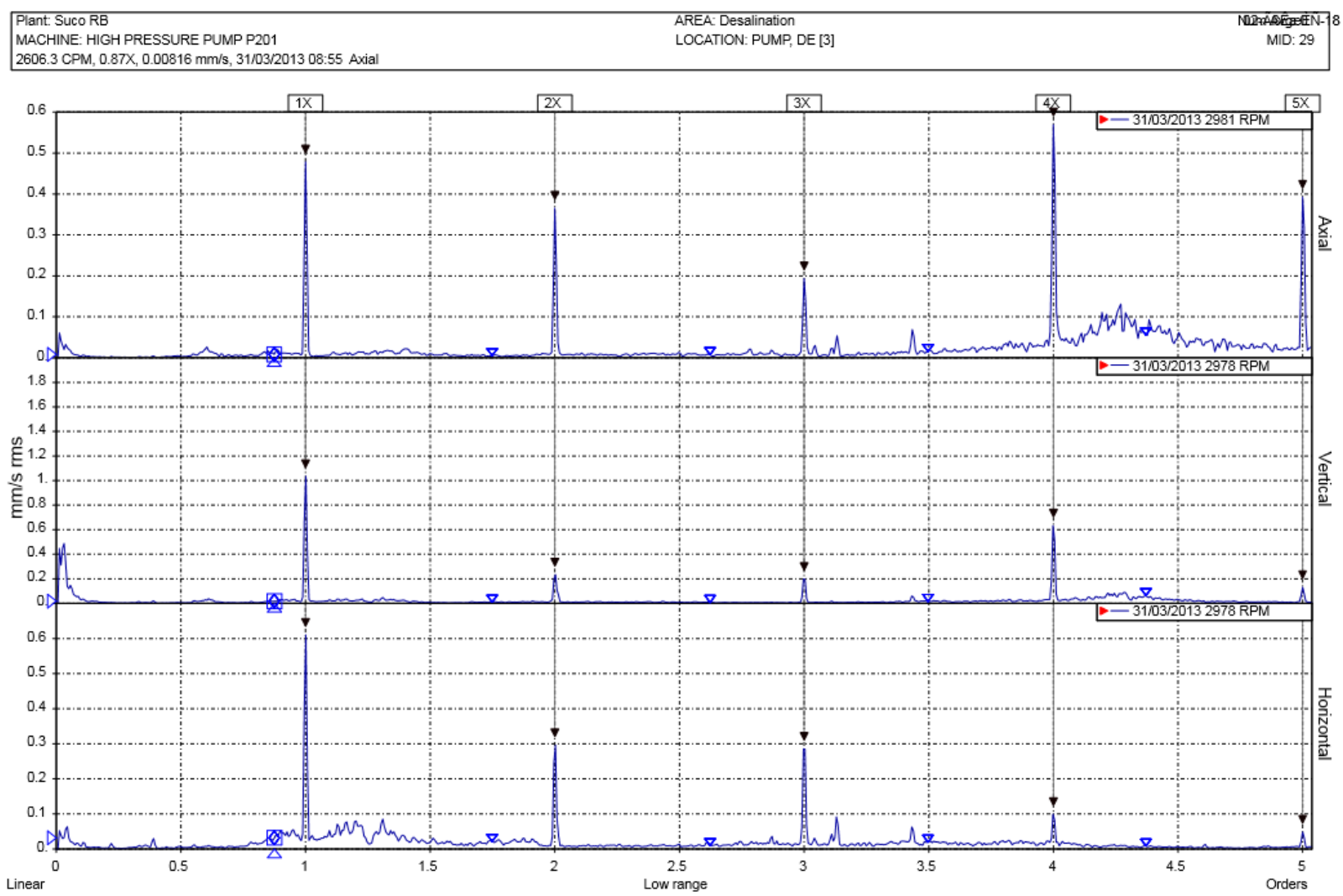

Fig. 8: Normal vibration signature of pump drive end

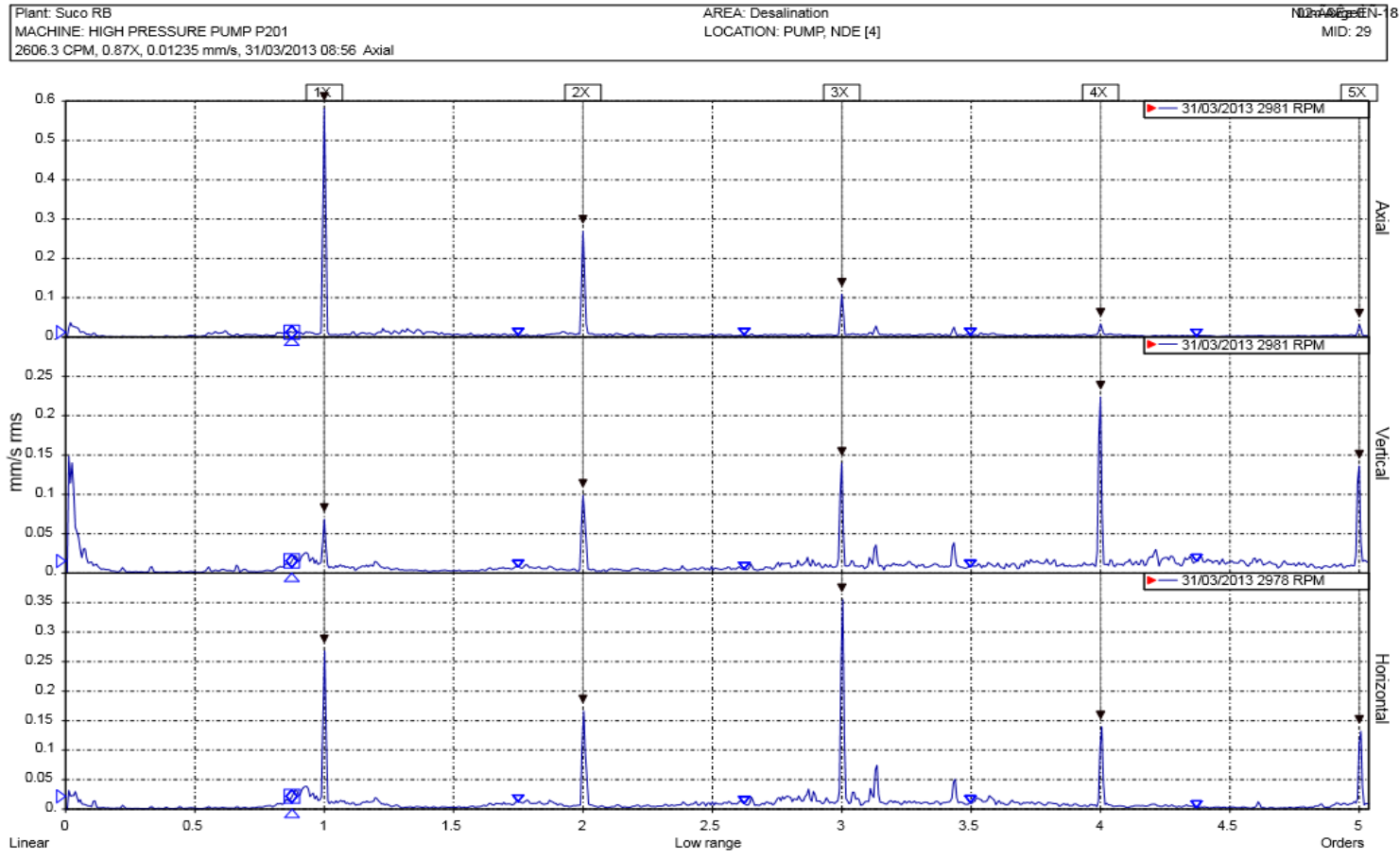

Fig. 9: Normal vibration signature of pump non-drive end 


\subsection{Faulty condition}

The frequency signatures of the faulty pump are shown in Figures 10 through 13. The vibration sensor measures the vibration at three directions for the same pump and motor's bearings. Table 2 shows the measured amplitude reading of the abnormal operation at the four positions of the pumping system at the three axes. The readings are compared with the alarm and critical limits.

Figure 10 shows the spectrum of the motor DE. It's observably that there is a noticeable increase in the amplitudes of $1 \mathrm{X}=(4.85 \mathrm{~mm} / \mathrm{s}$ at axial, $2.6 \mathrm{~mm} / \mathrm{s}$ at vertical and $3.5 \mathrm{~mm} / \mathrm{s}$ at horizontal).
Figure 11 shows the spectrum of the motor It's obvious that there is extreme rise in the amplitude of $1 \mathrm{X}=(5.4 \mathrm{~mm} / \mathrm{s}$ at axial, $4.02 \mathrm{~mm} / \mathrm{s}$ at vertical and $4.2 \mathrm{~mm} / \mathrm{s}$ at horizontal).

Figure 12 shows the spectrum of the pump DE. It's observed that there is extreme rise in the amplitude of $1 \mathrm{X}=(1.3 \mathrm{~mm} / \mathrm{s}$ at axial, $2.7 \mathrm{~mm} / \mathrm{s}$ at vertical and 3.2 $\mathrm{mm} / \mathrm{s}$ at horizontal).

Figure 13 shows the spectrum of the pump NDE. It's obvious that there is extreme rise in the amplitude of $1 \mathrm{X}=(1.39 \mathrm{~mm} / \mathrm{s}$ at axial, $0.75 \mathrm{~mm} / \mathrm{s}$ at vertical and $1.7 \mathrm{~mm} / \mathrm{s}$ at horizontal). There is a noticeable change in the readings and it's exceeding the alarm limits.

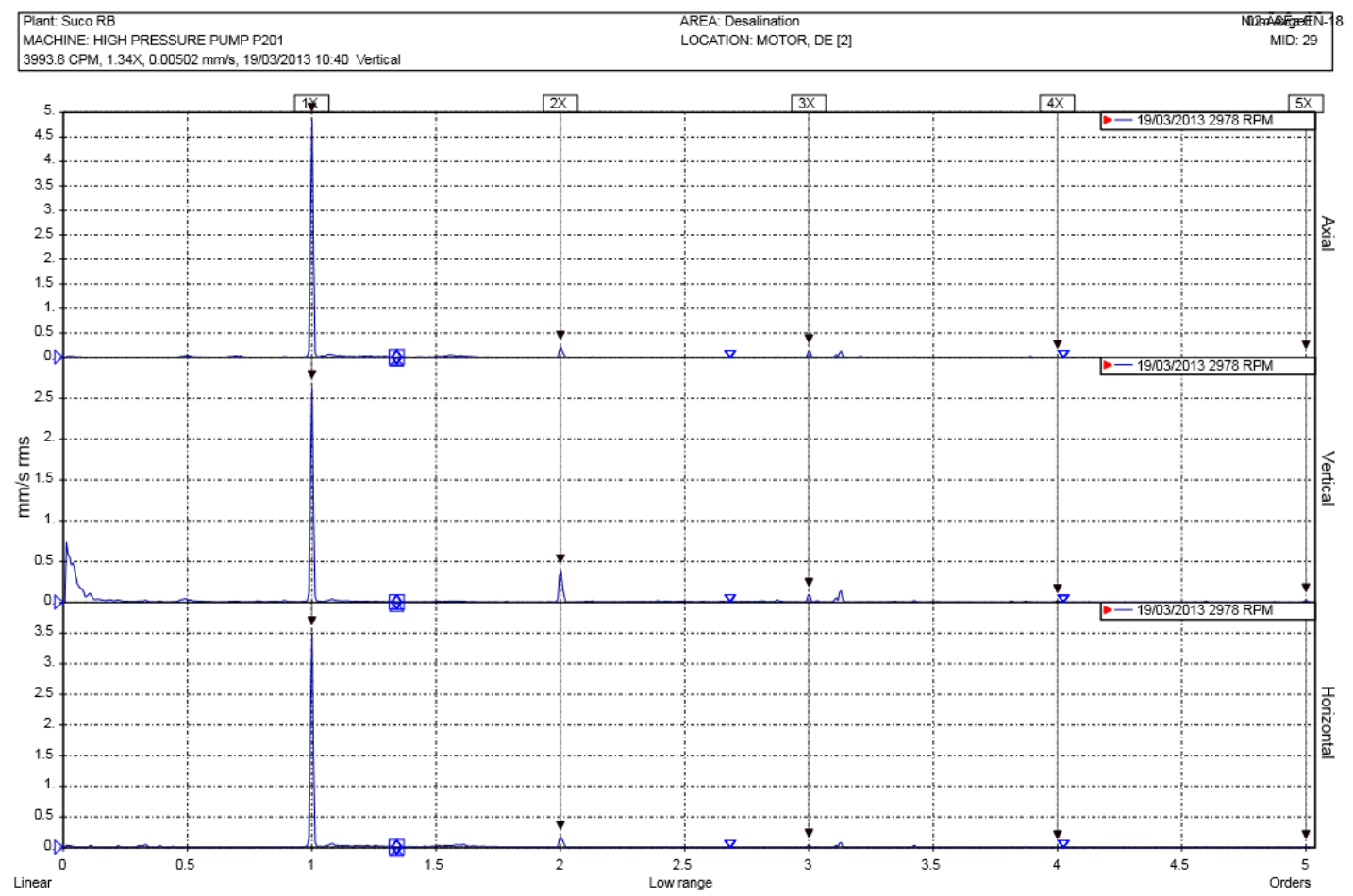

Fig. 10: Abnormal vibration signature of motor drive end due to unbalanced rotor 


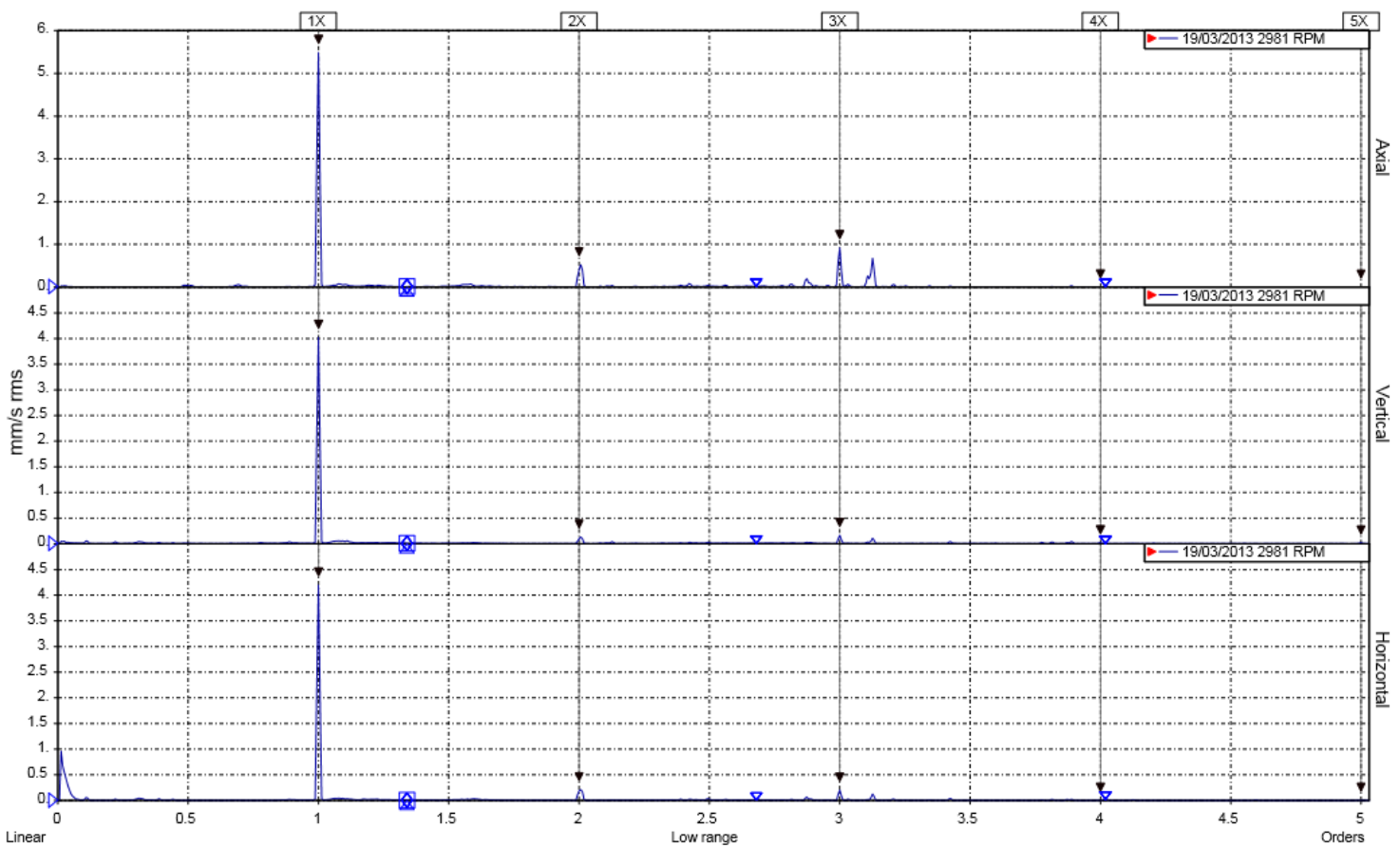

Fig. 11: Abnormal vibration signature of motor non-drive end due to unbalanced rotor

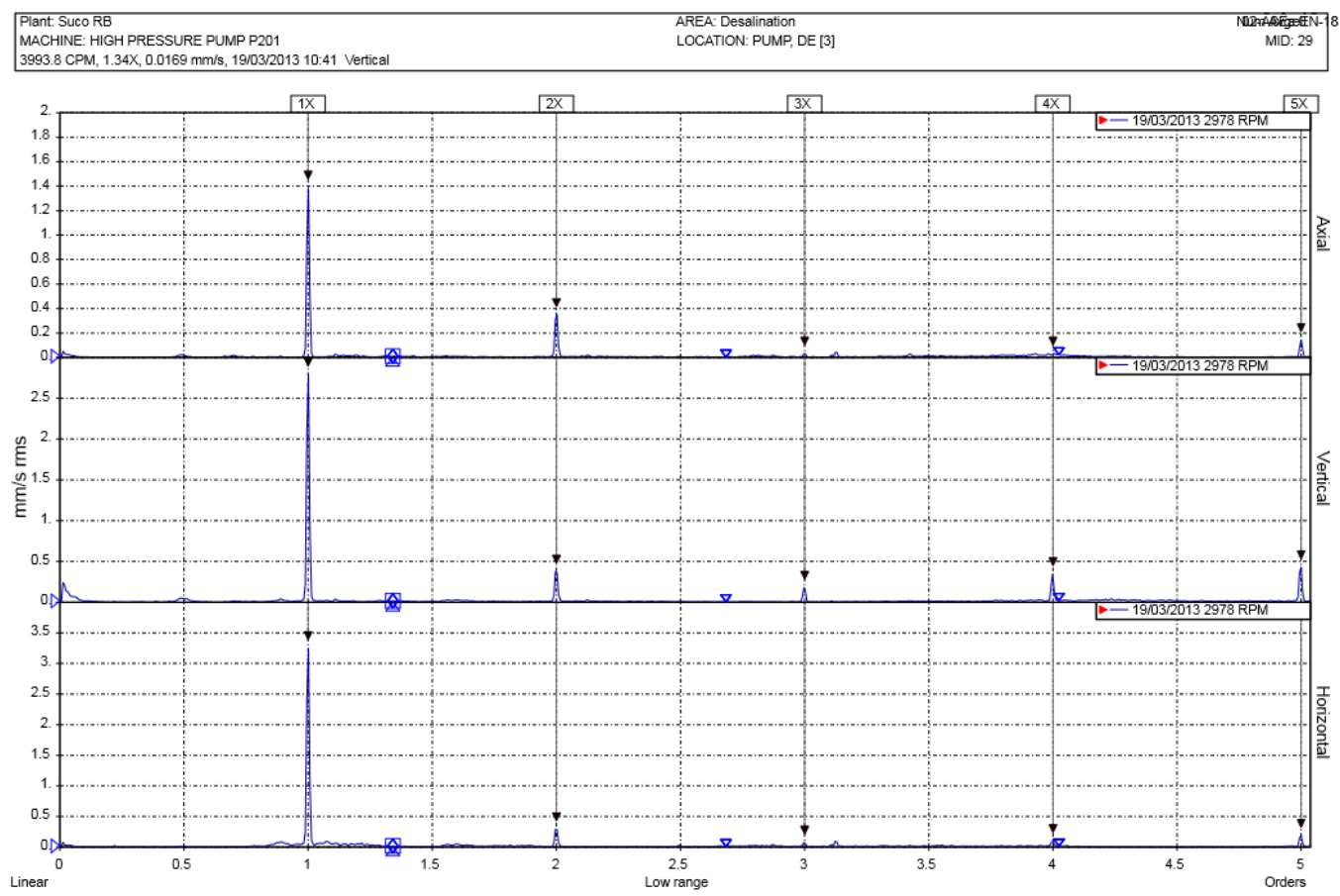

Fig. 12: Abnormal vibration signature of pump drive end due to unbalancedrotor 


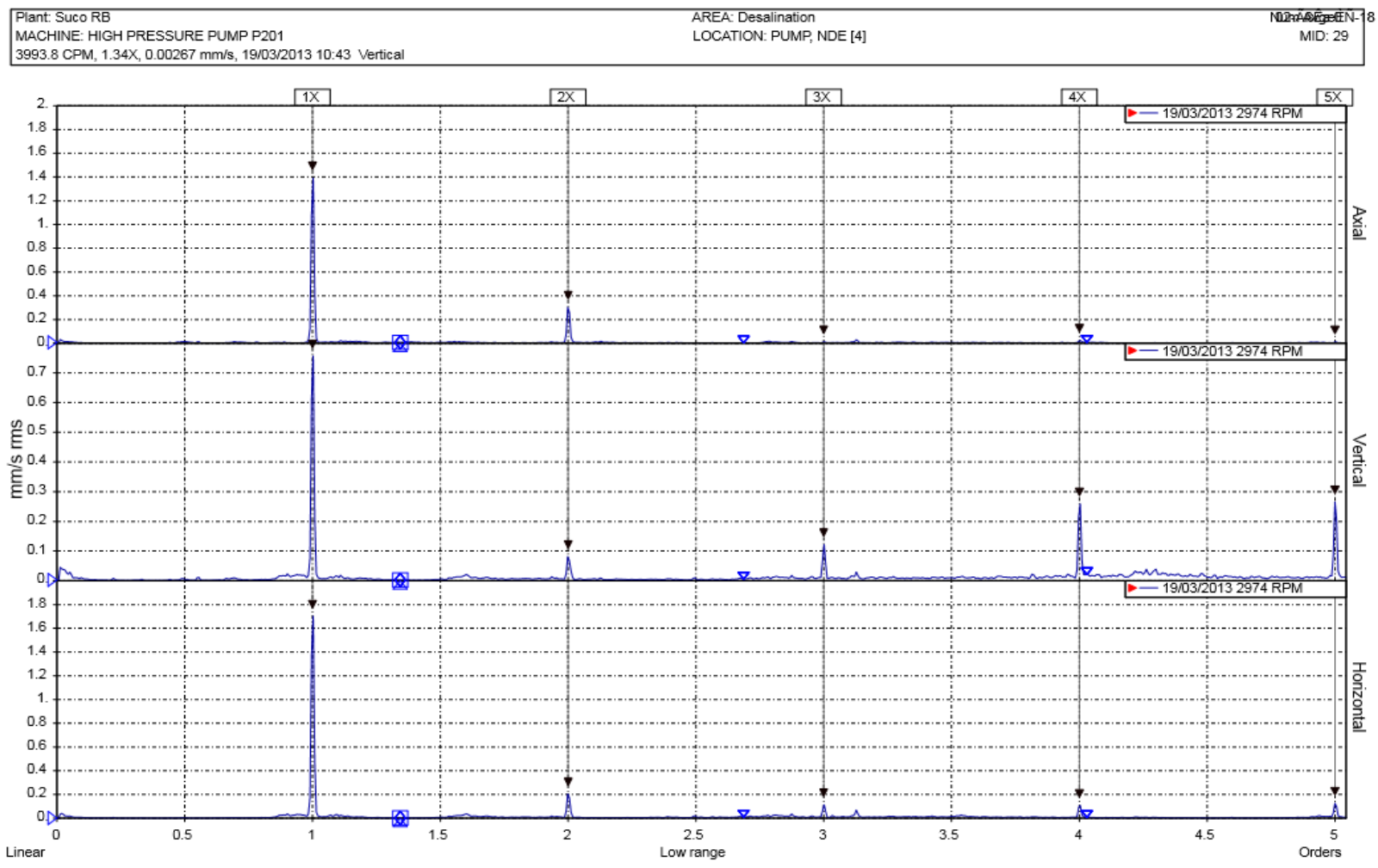

Fig. 13: Abnormal vibration signature of pump non-drive end due to unbalancedrotor

Comparison between the normal and abnormal overhaul reading

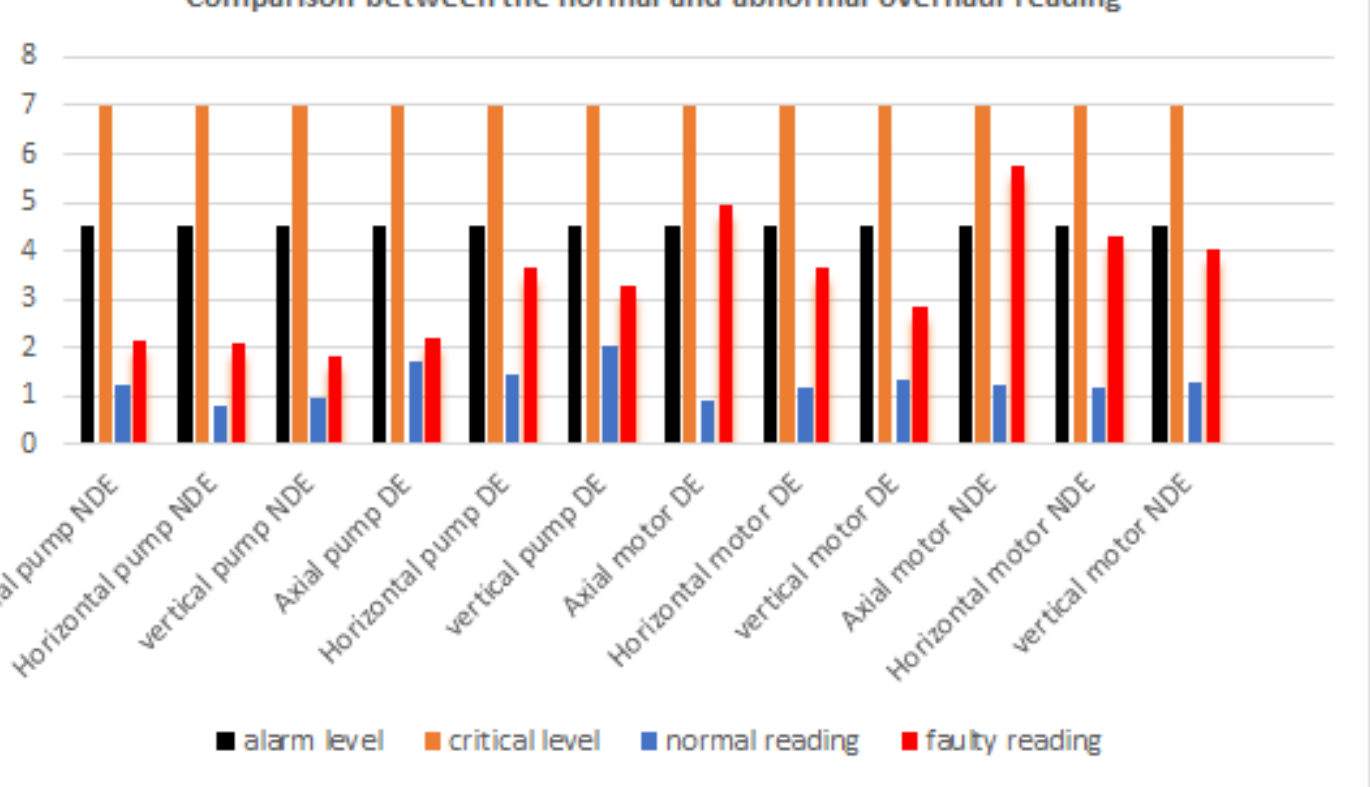

Fig. 14: Comparison between the normal and abnormal (due to unbalance in the rotor) overall reading from Table 1 
5.3 Comparison between normal and abnormal faulty conditions due to unbalance in the rotor

Figure 14 shows the comparison between the alarm, critical level, overall readings and the abnormal readings in sequence.

The comparison between the normal and faulty conditions are shown on Figures 15 through 18, where the faulty case is represented with black color and the normal case is represented with blue color at the four positions. There is an increase in the amplitude of the abnormal reading than the normal reading at $1 \mathrm{X}$.

Figure 19 shows the time waveform of the motor drive end at the three axes. The $\mathrm{X}$ axis represents the time in second and the $\mathrm{Y}$ axis represents the amplitude in $\mathrm{mm} / \mathrm{s}$. it is obvious that the wave is sinusoidal wave. Figure 20 shows the time waveform of the motor non-drive end at the three axes

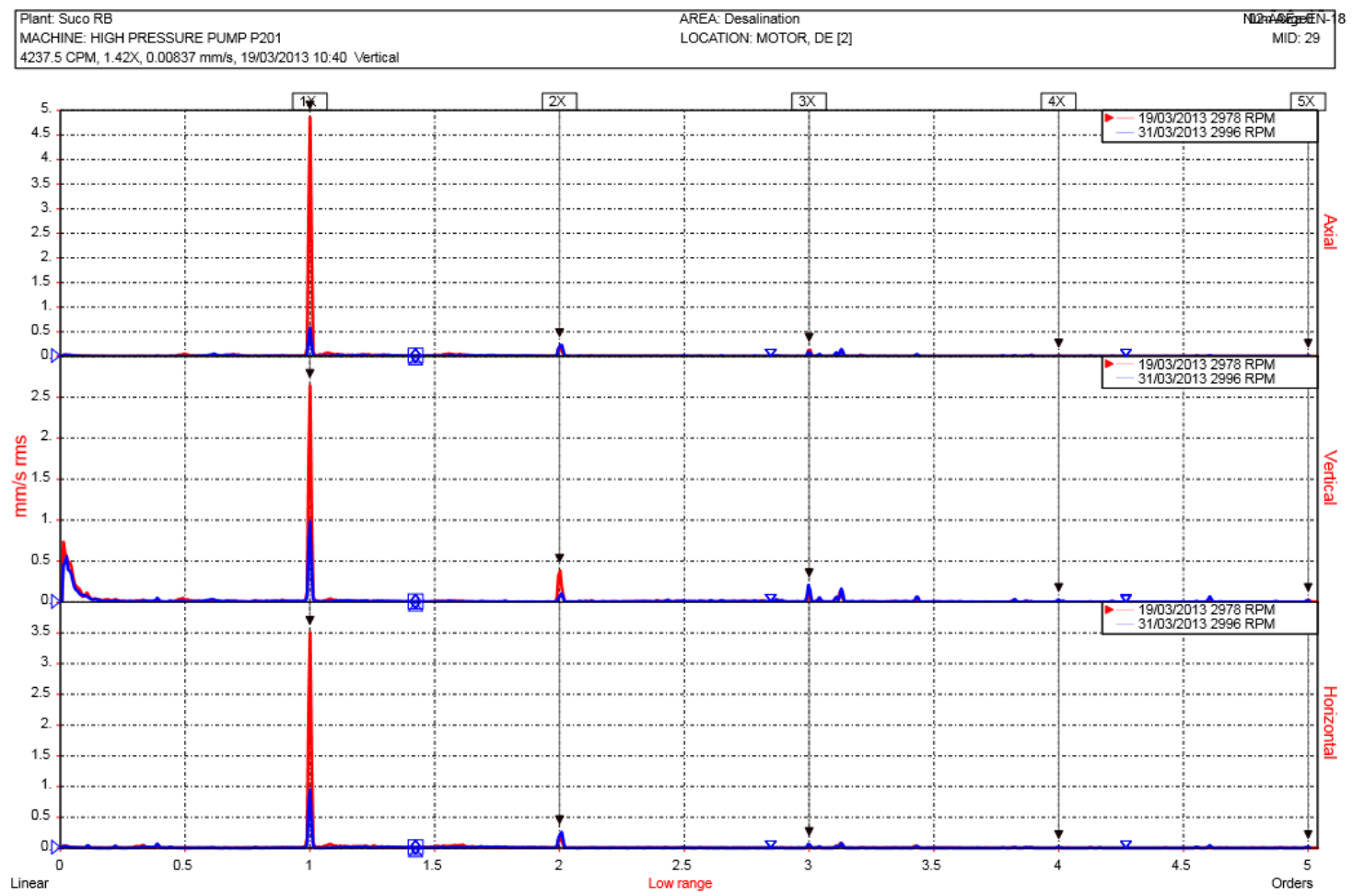

Fig. 15: Comparison between normal and abnormal vibration signature of motor drive end due to unbalance 


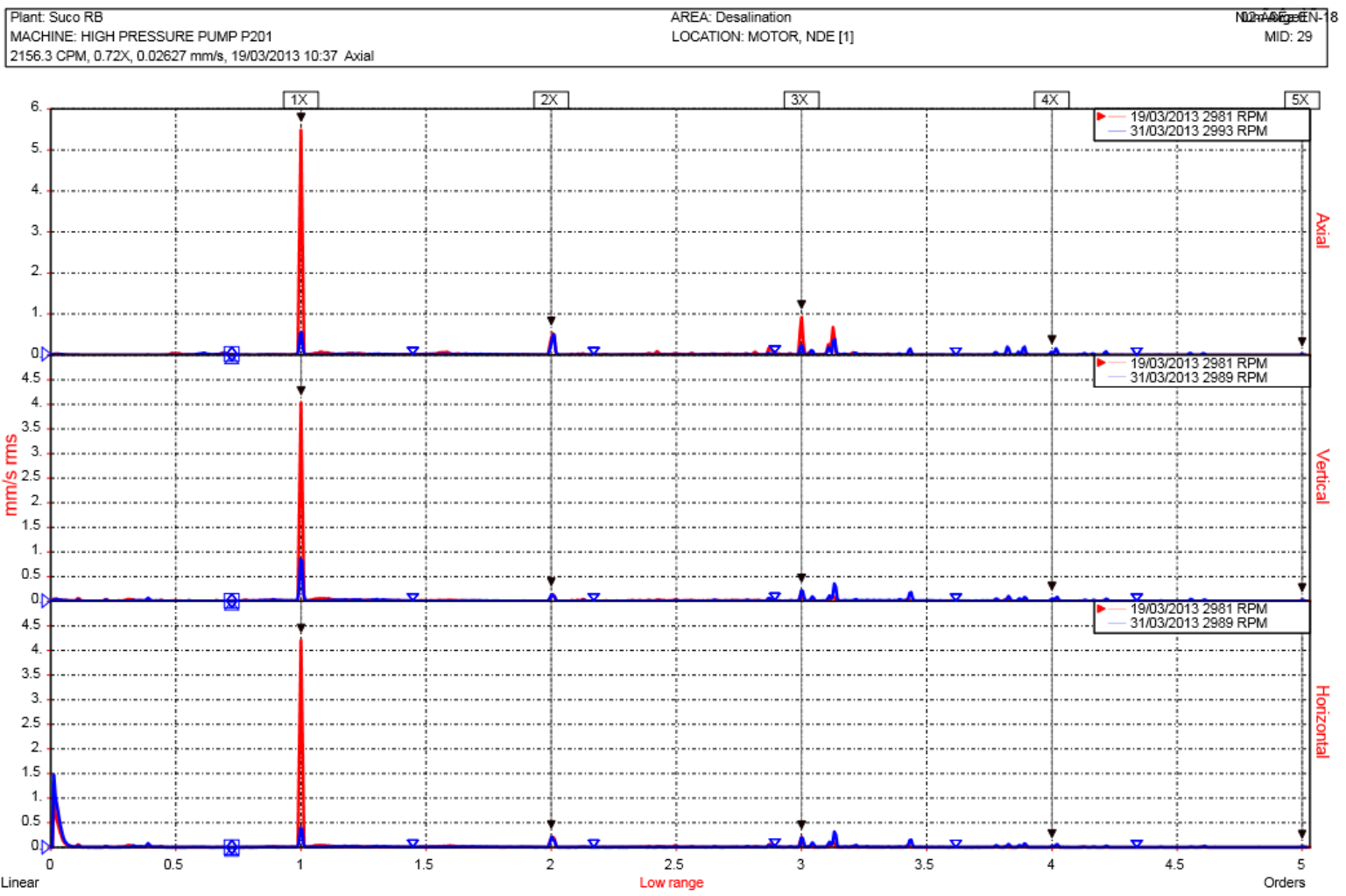

Fig. 16: Comparison between normal and faulty vibration signature of motor non- drive end due to unbalance

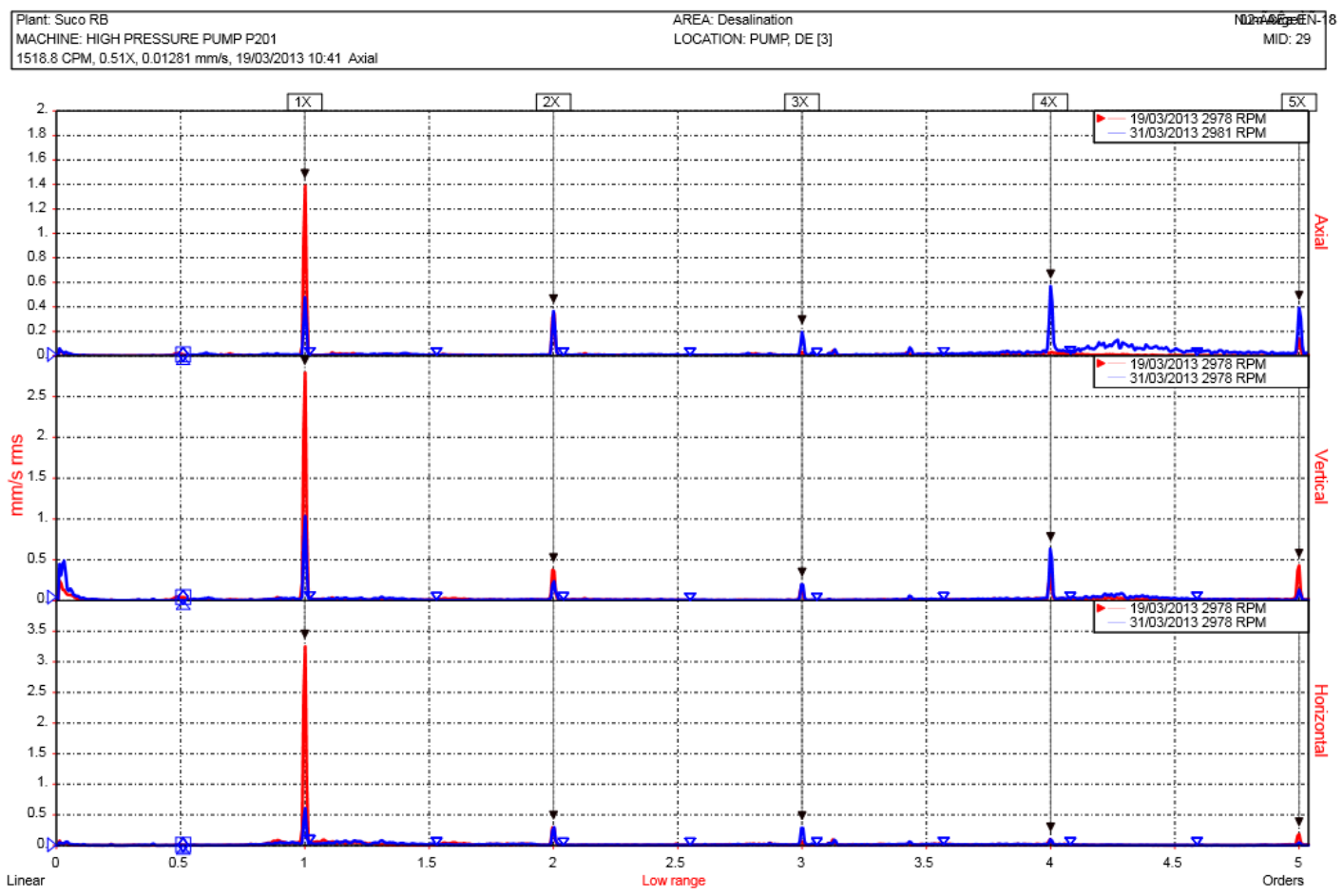

Fig. 17: Comparison between normal and faulty vibration signature of pump drive end due to unbalance 
Ahmed H. Osman et al. / Vibration Signature of Unbalanced Rotors of Centrifugal Pumps

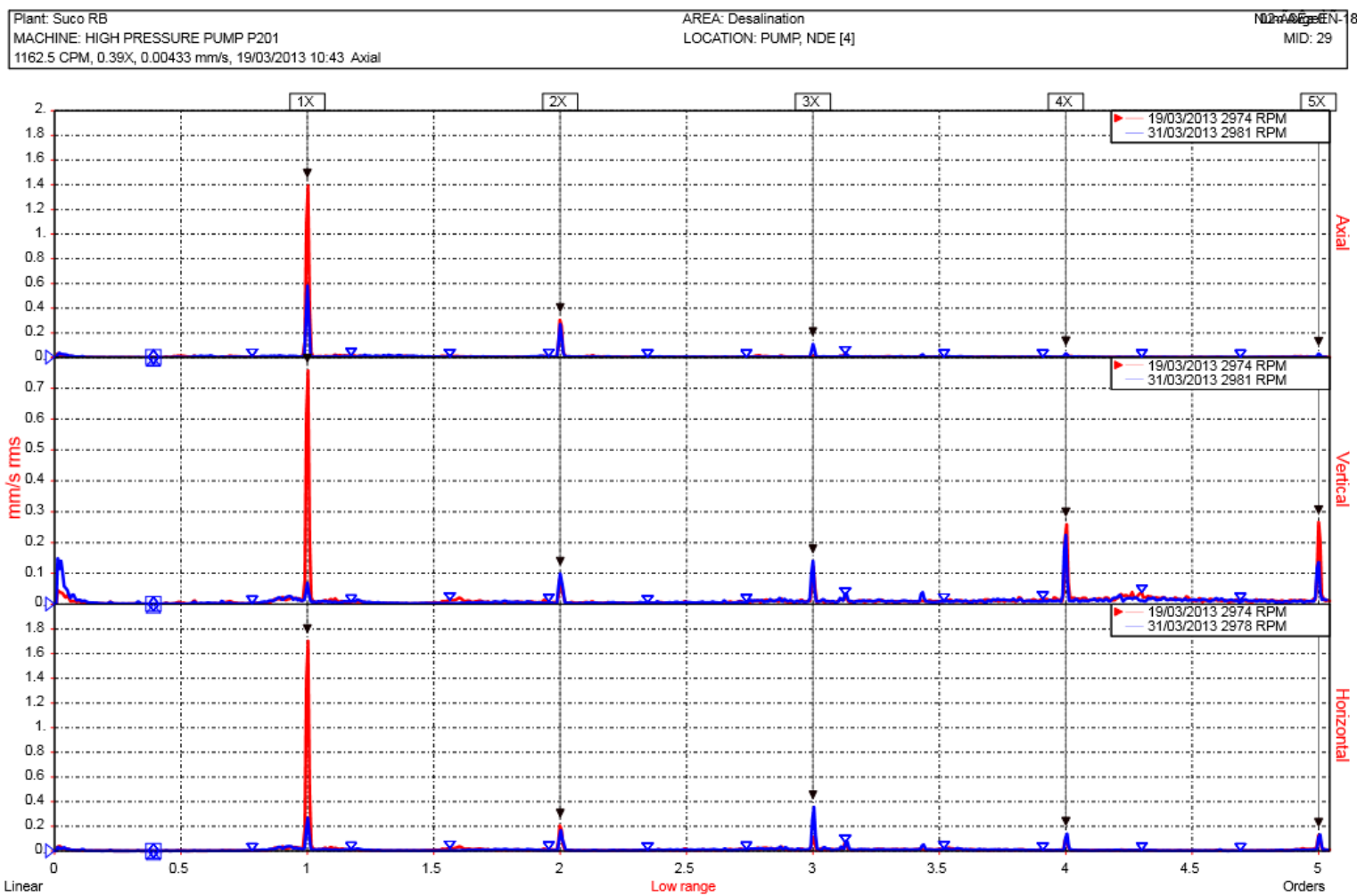

Fig. 18: Comparison between normal and faulty vibration signature of pump non-drive end due to unbalance

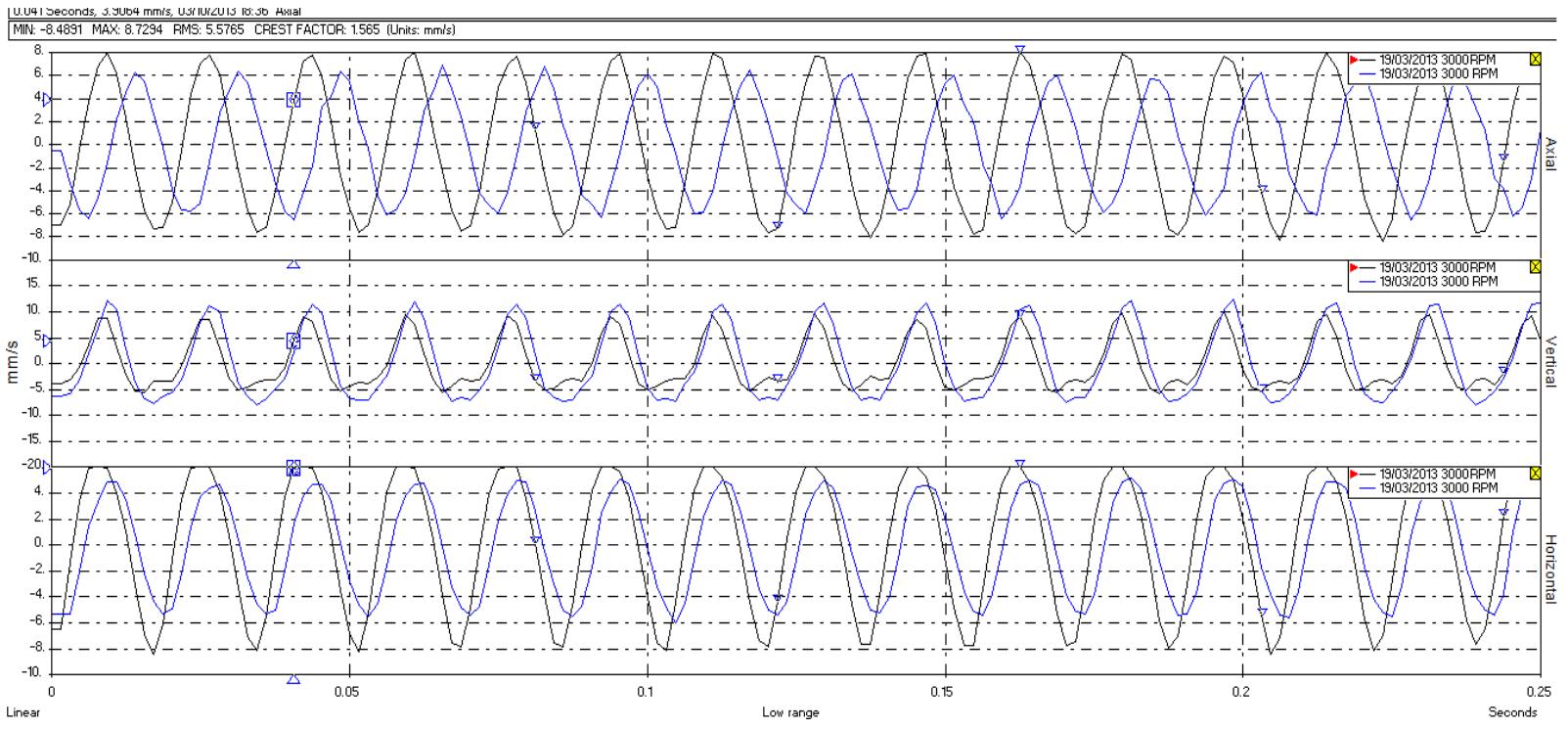

Fig. 19: Motor bearings (drive end \& non-drive end) are in-phase 


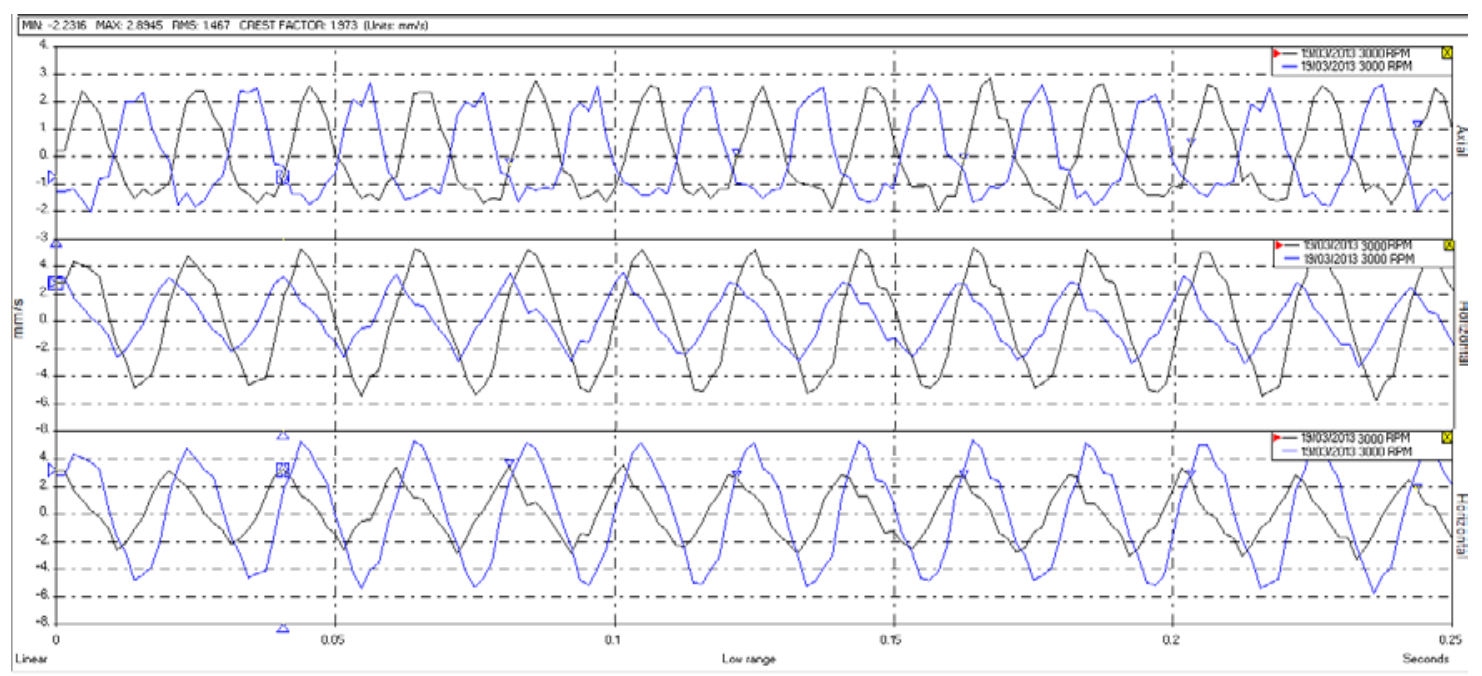

Fig. 20: Pump bearings (drive end \& non-drive end) are in-phase

\section{Finding and discussion}

Vibration signatures, as shown in Figures 15, 16, 17, and 18 show the maximum average amplitudes for all positions (Motor DE, Motor NDE, Pump DE and Pump NDE) in three directions (A, $\mathrm{V}$ and $\mathrm{H}$ ). The maximum amplitude exists at $1 \mathrm{X}$ RPM $(\approx 50 \mathrm{~Hz})$. The vibration spectrum is represented for both cases (normal and faulty due to unbalance) and for all positions (Motor DE, Motor NDE, Pump DE and Pump NDE). It is found that the average maximum amplitude at $1 \mathrm{X}$ in the three directions for faulty condition is higher than the normal condition. It has obviously seen that the amplitudes (of the motorDE

5.74 \& motor NDE 4.97) in the axial direction represent the highest amplitudes because the unbalance creates a bending moment on the shaft causing the bearing house to move axially. The amplitudes at $1 \mathrm{X}$ levels are nearly equal when comparing the vertical and horizontal amplitudes. The amplitude increases proportionally with the square of the rotationalspeed.

Figure 19 shows a comparison between the motor bearings (DE \& NDE). This comparison indicates that there is a ninety $90^{\circ}$ phase shift in the axial direction but the vertical and horizontal direction are in phase. The time wave shows nearly sinusoidal waves. The noise on the measured signals may cause some deformation of the sinusoidal wave. This deformation may be corrected using a digital filter.

Unknown faults may also cause dditional deformation of the timewave
Figure 20 shows comparison between the pump bearings (DE \& NDE). This comparison indicates that there is a ninety $90^{\circ}$ phase shift in the axial direction but the vertical and horizontal direction are in phase. This case may be considered as a good example for overhung unbalance

\section{Conclusion}

- The experimental study shows that the unbalanced rotors (in an overhung or cantilevered pump) generates a high amplitude peak at $1 \mathrm{X}$ of shaft rotational speed when measured in the radial direction. The vibration level at the axial direction will be highest.

- Unbalance produces a bending moment on the shaft causing the bearing house to move axially leading to increase the amplitude in this direction.

- For the same unbalanced weight, increasing the rotational speed results in increasing the amplitude at $1 X$. Actually, the increase in the centrifugal force due to increasing the speed increases the amplitude value. The amplitude increases proportionally with the square of the rotational speed.

- By comparing the vertical and the horizontal $1 \mathrm{X}$ level found that they are nearly equal.

- It may be necessary to break the coupling 
between the pump and the motor to know which of them is the cause of unbalance. Monitoring the amplitude at $1 \mathrm{X}$ for the motor will indicates whether it's the cause of unbalance or thepump.

- The time domain signal has a sinusoidal wave. The existence of some noise or unknown faults may slightly deform the sinusoidalwave.

- 1X RPM peaks should occur on both bearingsin the same direction.

- $1 X$ RPM peaks in the vertical direction are greater than the $1 \mathrm{X}$ RPM peaks in the horizontal direction

\section{Reference}

[1] Marius STAN, “On the Durability of Centrifugal Pumps”, FiabilitatesiDurabilitate - Fiability\&Durability No 1/ 2018 TârguJiu, ISSN 1844 - 640X

[2] R. Kiliç , "Determination of Imbalance Problem in Electric Motor and Centrifugal Pump by Vibration Analysis”, ACTA PHYSICA POLONICA A, Vol. 130 ( July, 2016). PP. 487- 492.

[3] K.P. Ramachandran, "Vibration signature analysis for machine health monitoring and fault diagnosis", Caledonian Journal of Engineering, PP. 26-39,2004.
[4] Surendra N. Ganeriwala\& Brian Schwarz \& Mark H. Richardson, "Usingoperating deflection shapes to detect unbalance in rotating equipment", Spectra QuestInc, Inc Vibrant Technology, Vol. 43(5), PP. 1113, 2009.

[5] V. Hariharan and P.S.S. Srinivasan, "Vibrational Analysis of Flexible Coupling by Considering Unbalance", World Applied Sciences Journal, Vol. 8 (8), PP. 1022-1031, 2010.

[6] M. Abdul Saleem, G. Diwakar, Dr. M.R.S. Satyanarayana, "Detection of unbalance in rotating machines using shaft deflection measurement during its operation", Journal of Mechanical and Civil Engineering (IOSR- JMCE), Vol.3 (3), PP. 08-20,2012.

[7] Girish D. Mehta, Vijaykumar S. Shende,Prerna S. Borkar, "A mathematical model for vibration-based prognosis for effect of unbalance on journal bearing", International Journal of Engineering Research and Applications (IJERA), Vol. 3(1), PP. 537-544, 2013.

[8] http://www.kittiwakeholroyd.com /vm-iso- 10816.htm

[9] PareshGirdha, Cornelius Scheffer, "Practical machinery vibration analysis and predictive maintenance", Newnes an imprint of Elsevier, IDC Technologies,2004

\begin{tabular}{|c|c|c|c|c|c|c|}
\hline \multicolumn{7}{|c|}{ VIBRATION SEVERITY PER ISO 10816} \\
\hline \multicolumn{3}{|c|}{ Machine } & \multirow{2}{*}{$\begin{array}{l}\text { Class I } \\
\text { small } \\
\text { machines }\end{array}$} & \multirow{2}{*}{$\begin{array}{l}\text { Class II } \\
\text { medium } \\
\text { machines }\end{array}$} & \multirow{2}{*}{$\begin{array}{l}\text { Class III } \\
\text { large rigid } \\
\text { foundation }\end{array}$} & \multirow{2}{*}{$\begin{array}{l}\text { Class IV } \\
\text { large soft } \\
\text { foundation }\end{array}$} \\
\hline & $\mathrm{in} / \mathrm{s}$ & $\mathrm{mm} / \mathrm{s}$ & & & & \\
\hline \multirow{4}{*}{$\stackrel{\mathscr{n}}{5}^{\infty}$} & 0.01 & 0.28 & & & & \\
\hline & 0.02 & 0.45 & & & & \\
\hline & 0.03 & 0.71 & & \multicolumn{2}{|c|}{ good } & \\
\hline & 0.04 & 1.12 & & & & \\
\hline \multirow{8}{*}{ 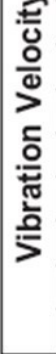 } & 0.07 & 1.80 & & & & \\
\hline & 0.11 & 2.80 & & \multicolumn{2}{|c|}{ satisfactory } & \\
\hline & 0.18 & 4.50 & & & & \\
\hline & 0.28 & 7.10 & & \multicolumn{2}{|c|}{ unsatisfactory } & \\
\hline & 0.44 & 11.2 & & & & \\
\hline & 0.70 & 18.0 & & & & \\
\hline & 0.71 & 28.0 & & \multicolumn{2}{|c|}{ unacceptable } & \\
\hline & 1.10 & 45.0 & & & & \\
\hline
\end{tabular}

